\title{
Intelligent Protocol Analyzer for WWW Server Accesses with Exception Handling Function
}

Tomohiko Ogishi, Akira Idoue, Toshihiko Kato and Kenji Suzuki KDD R\&D Laboratories, Inc. 2-1-15, Ohara, Kamifukuoka-shi, Saitama 356, Japan E-mail : \{ogishi, idoue, kato, suzuki\}@hsc.lab.kdd.co.jp

\begin{abstract}
Recently, the TCP/IP protocols are widely used and it is mentioned that there are problems such that the throughput is limited due to protocol procedures such as retransmission and congestion control of TCP. In order to analyze these problems, we have developed an "intelligent" protocol analyzer which can estimate what communication has taken place by emulating the behaviors of the IP, TCP and HTTP protocol entities, which are used for WWW server access communications. This analyzer also supports the handling of exceptional situations such as monitoring errors and network misbehaviors. This paper describes the details of the emulation functions of HTTP and the exception handing functions, and shows some results of applying the analyzer to actual WWW server accesses.
\end{abstract}

Keywords

Protocol Analyzer, HTTP, WWW, HTML, TCP

\section{INTRODUCTION}

Recently, the TCP/IP protocols $[1,2]$ are widely used in various computer communications. Here, most users of computers use communication functions 
installed in operating systems or commercial software products as they are, and do not pay any attentions to their details. However, some problems occur for TCP/IP communications, in the cases that there are some packet losses due to network congestion and transmission errors, and that the protocol parameters of communicating computers are not matched.

In order to analyze those problems, we developed an "intelligent" protocol analyzer for TCP (Transmission Control Protocol), which can estimate what communication has taken place among communicating computers [3, 4]. This analyzer maintains the specification of the state transition based behaviors of TCP and emulates the behaviors of the TCP protocol entities in communicating computers. Since modern TCP includes some internal procedures for the flow control, such as the slow start algorithm, this analyzer can emulate these procedures as well. This analyzer provides information such as mappings between data segments and ack segments and updates of congestion window used for the congestion control, and this releases the burden of analyzing TCP behaviors much better than the commercially available protocol monitors [5].

However, our intelligent protocol analyzer has two points to be improved. The first one is that, since it focuses only on protocols up to TCP, it is required to implement the analyzing functions of application protocol for the examination of actual communications. Recently, WWW server access is considered to be the most common application, and here data of Web pages are transferred according to HTTP (Hyper Text Transfer Protocol) [6] located on top of TCP. Since one Web page consists of several elements to be retrieved through different TCP connections, the related data transfers to one Web page need to be combined in the examination of the Web page access. For this purpose, the application protocols, HTTP and HTML (Hyper Text Markup Language) in this case, need to be supported by our intelligent analyzer.

The second one is to add the functions to cope with exceptional situations such that the analyzer drops segments and the sequence of observed segments is not the same as that of sent out or received segments. In order to add these functions, the analyzer needs to estimate the possible situations if it detects some errors in observed segment sequence.

We have implemented new version of intelligent protocol analyzer which supports the analysis of IP, TCP and HTTP, which is the protocol stack for WWW server access application, and can handle exceptional situations such as monitoring errors and network misbehaviors. This paper describes the design of our new analyzer, which we call the $W W W$ intelligent protocol analyzer, and some results applying it to actual communications. The next section describes the design overview of our WWW intelligent protocol analyzer. Sections 3 and 4 describes the detailed design of the emulation function of HTTP and the exception handling function, respectively. Section 5 gives some results of applying our analyzer to actual WWW server access communications. 


\section{DESIGN OVERVIEW}

(1) As depicted in Fig. 1, the WWW intelligent protocol analyzer is attached to a LAN, and captures and stores all PDUs (Protocol Data Units) transmitted over the LAN. The analyzer selects PDUs transmitted or received by a specific computer focused on from the stored PDUs, and analyzes their formats and the behaviors of TCP and HTTP protocol entities in the specific computers and the computers communicating with it, e.g. computers $\mathrm{A}, \mathrm{B}$ and $\mathrm{C}$ in the figure.

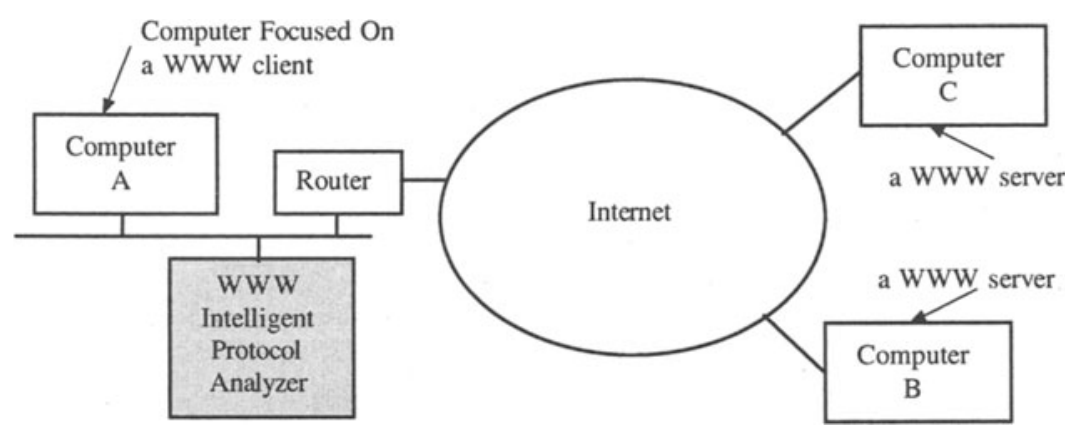

Figure 1 Configuration using WWW Intelligent Protocol Analyzer.

(2) Figure 2 depicts the software structure of the analyzer. The software is developed based on the previous intelligent protocol analyzer, and the modified or newly developed part is indicated in the figure.

- The PDU capture module captures PDUs transmitted over the LAN, analyzes their format and parameter values according to the IP, TCP and HTTP protocols, and saves those results in the monitor log.

- The event sequence estimation module selects PDUs which the computer focused on sent or received, and constructs the event sequence logs, which consist of a sequence of sent and received TCP segments and their timing, for individual computers involved in the communication by taking account of the transmission delay between the analyzer and the computers [3].

- The TCP emulation module emulates the TCP behaviors of these computers according to the event sequence logs. This module maintains the state transition specification of TCP for sent and received events, and estimates the state and variables in individual computers according the following procedure [3].

- For a received TCP segment, it looks up the specification for received events, and performs a corresponding state transition. If it sends out a segment, the module checks a sent TCP segment in the event sequence and emulates the received and sent segments.

- For a sent TCP segment, the TCP emulation module looks up the specification for sent events and checks whether the TCP protocol entity can send out the segment. 
- The HTTP emulation module, which is newly added, maintains the state transition specification of HTTP and emulates the HTTP behaviors in individual computers being examined.

(3) In order to realize the analysis of WWW server accesses, the following functions are developed.

- The PDU capture module stores the HTTP header information and the HTML text as well as the IP and TCP headers.

- The TCP emulation module reports to the HTTP emulation module the events such that a TCP connection is established or released, and HTTP data is sent or received. We call these events $T C P$ primitives. It needs to be mentioned that the TCP primitives are reported as the results of TCP processing and therefore the sequence of these primitives in a sending and receiving computers are the same even if some segments are lost and retransmitted by TCP.

- The HTTP emulation module provides the following functions;

- Emulation of HTTP behavior: This module maintains the state transition specification which we have introduced, and manages the state and variables of HTTP for individual TCP connections used for WWW server accesses.

- Web page link: As described above, one Web page consists of several elements such as HTML text and graphical image, and different TCP connections are used to retrieve these elements. One Web page also has several links to other Web pages, which may be accessed to using different TCP connections. Therefore, in order to analyze the communication for accessing one Web page, the HTTP emulation module links those Web page data accesses.

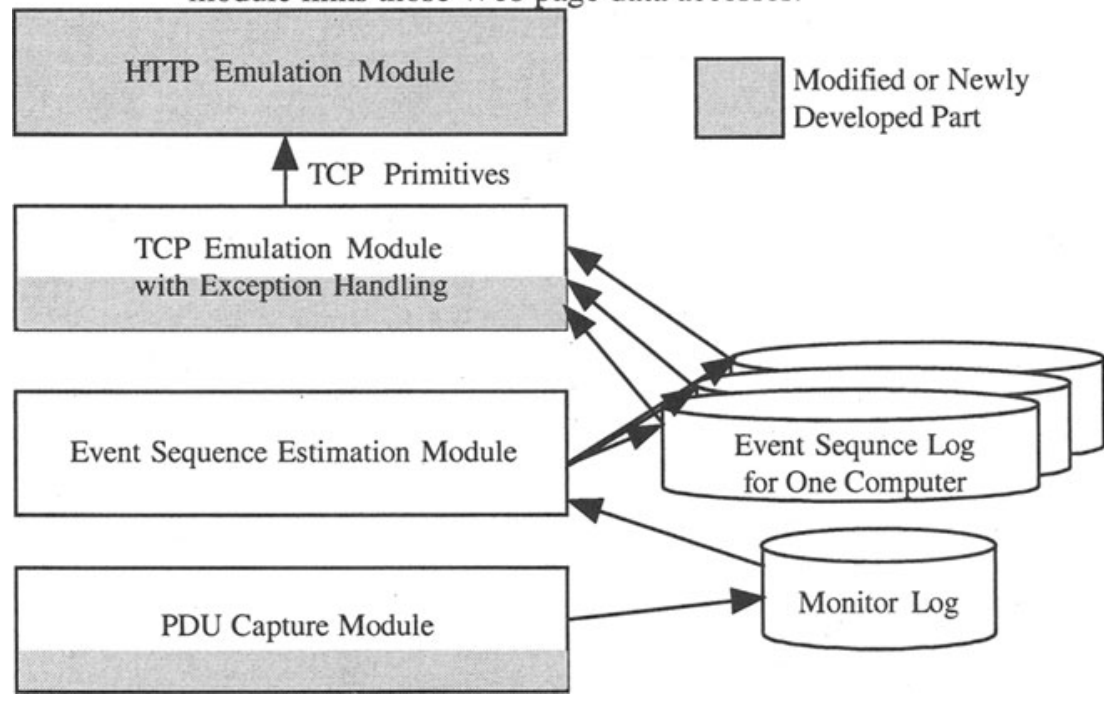

Figure 2 Software Structure of WWW Intelligent Protocol Analyzer 
(4) In order to realize the exception handling, we add the following functions in the TCP emulation modules.

- We take account of the following cases in which the sequence the analyzer captures are different from the real sequence handled in the computers being examined.

- Drop: The analyzer fails to capture PDUs.

- Timing error: As describe above, the analyzer estimates the processing timing of PDUs in individual computers according to the transmission delay, and therefore, it is possible to make wrong estimation.

- PDU losses at sender side: When a PDU is lost between the sending computer and the analyzer, the analyzer cannot observe it.

- PDU losses at receiver side: When a PDU is lost between the analyzer and the receiving computer, the receiving computer will not handle it although it is observed by the analyzer.

- Misordering in network: When the order of PDUs are changed in the network, the sequence observed by the analyzer is different from the real sequence in the computers.

- In those cases, it is possible that the TCP emulation module decides that the TCP entities in computers have some protocol errors. Here, the module checks whether the detected errors can be solved by assuming one of the above cases occurs. If it can be solved, the module handles that there are no protocol errors but there are exceptional situations.

\section{EMULATION FUNCTION FOR HTTP \\ 3.1. Emulation of HTTP Behavior}

The HTTP procedure uses an HTTP request message in order to specify the identifier of a Web page and uses an HTTP response message in order to respond to the request [6]. Each HTTP request / response message consists of the header part including URI (Universal Resource Identifier), content type and content length, and the body part including the content like a HTML text or a graphical image. If its content length is longer than MSS (Maximum Segment Size), the content would be divided into several TCP segments.

In order to emulate these behaviors of HTTP entities, we introduce the HTTP state transition, a part of which is depicted in Fig. 2. The inputs of the state transition are TCP primitives which we defined. They includes the followings.

CNREQ : issue of TCP connection establishment request

CNIND : receipt of TCP connection establishment request

CNCNF : completion of TCP connection establishment

DTSND : issue of data segment

DTRCV : receipt of data segment

CLOSE : release of TCP connection

The states of the HTTP state transition include the followings. 


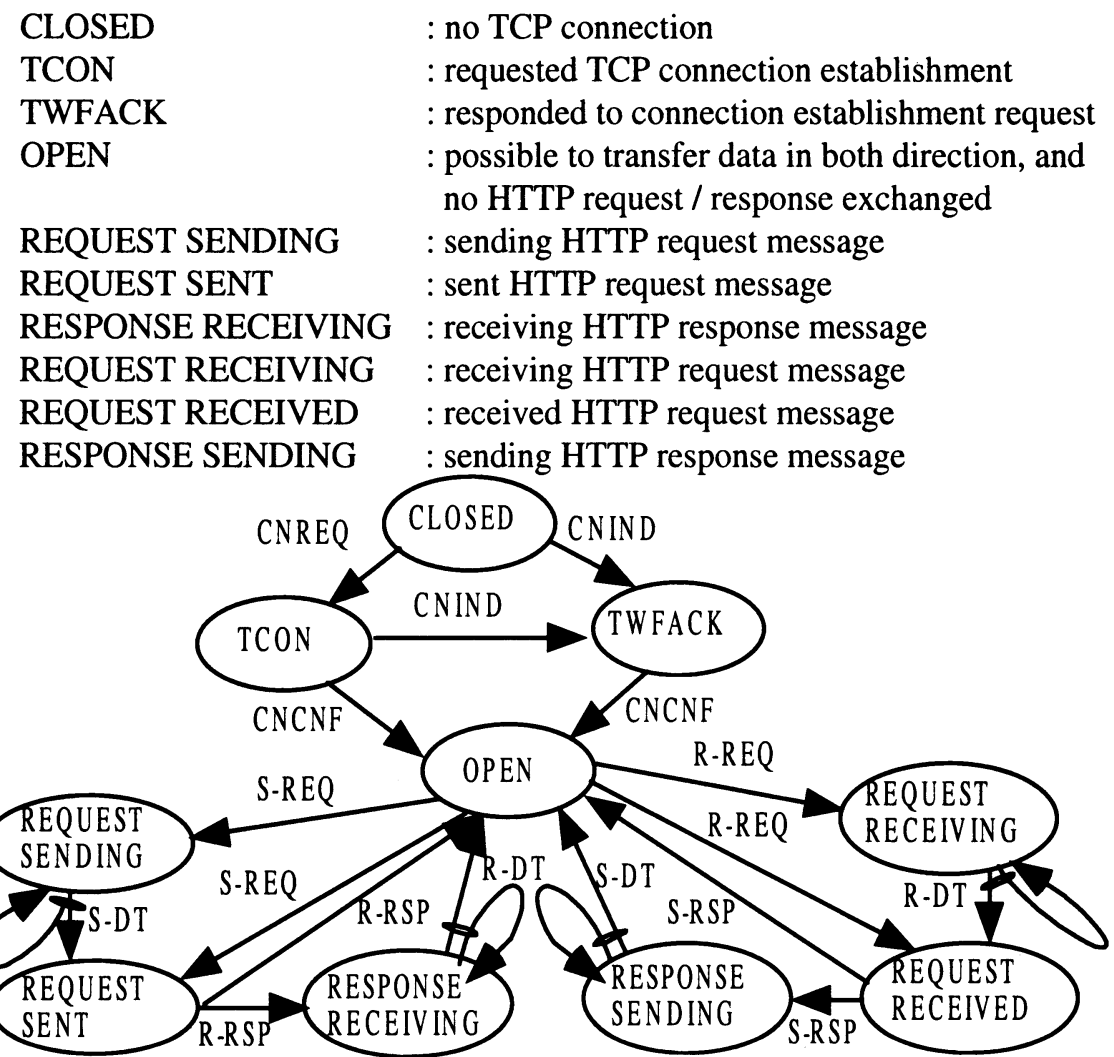

S-REQ: DTSND with REQ, S-RSP: DTSND with RSP, S-DT : DTSND without REQ/RSP

R-REQ: DTRCV with REQ, R-RSP: DTRCV with RSP, R-DT : DTRCV without REQ/RSP

$\left({ }^{*}\right)$ n receiving CLOSE event, state will change to CLOSED in all HTTP states

Figure 3 HTTP state transition

\subsection{Estimation of Web Page Link}

As described above, multiple pairs of requests and responses have link relationship in one Web page access. There are two types of these links. One is the case that a Web page contains more than one elements, and they are retrieved continuously. An example is that an HTML text contains keywords " $<$ IMG SRC=" or " $\angle$ FRAME $\mathrm{SRC}=$ " and we call this case an active link. The other is the case a Web page contains a hyper link to another page, e.g. an HTML text contains a keyword " $<$ A $\mathrm{HREF}="$, which we call a passive link.

In order to estimate the Web page link, we introduce the session ID and parent session ID, which are the identification of HTTP request and that of parent HTTP request, respectively. The estimation is performed in the following way.

(1) If an HTTP request message is new message for a Web page access, the HTTP emulation module assigns a session ID to the request message and 
remember a URL (Universal Resource Locator) included in the message header when it deals with a S-REQ (DTSND primitive with REQ).

(2) When the module deals with the response message corresponding to the request message and the content type is an HTML text, it searches keywords representing active link and passive link.

(3) When it finds an active link, it considers the retrieval of the Web page element as a child session of the current one. In this case, the HTTP emulation module assigns the child session ID and registers in the Web page link table a set of URL of the Web page element to be accessed, the session ID and the parent session ID. The child session ID is 1-1, 1-2 and so on if the parent session ID is 1 .

(4) When the module find a passive link, it does not assign session ID but registers URL and the parent session ID.

(5) When it deals with a new S-REQ, it checks if the URL of the request message exists in the Web page link table. When the URL is found as an active link, it gives the session ID registered in the table. When the URL is found as a passive link, it assigns a new session ID. If there are no URL in the table, the module considers that the request message is for a new Web page access.

\section{EXCEPTION HANDLING FUNCTION}

As described in section 2, the TCP emulation module will check the possibility of monitoring errors and network misbehaviors, i.e. drop, timing error, PDU losses at sender or receiver sides, and misordering, if it detects any protocol errors. If the protocol errors are solved by assuming that any of them have occurred, then the TCP emulating module considered that there are no protocol errors. The design of the exception handling function is performed for individual protocol errors. The following gives the details of some cases supported currently.

\section{(1) Sending SYN+ACK segment in state CLOSED}

In this case, there are two possibilities as depicted in Fig. 4 (1). One is the case that the analyzer estimated "SYN+ACK sent" has occurred prior to "SYN received." The other is the case that the analyzer dropped the SYN segment. In both cases, the actual sequence is that the SYN segment is received and the SYN+ACK segment is sent in response to it. Therefore, when the TCP emulation module detected this protocol error, it will search "SYN received" event. If it is found, the module considers that there are timing error, and otherwise, the module considers that it dropped a SYN segment.

(2) Receiving SYN segment in state CLOSED and sending no response In this case, there are two possibilities as depicted in Fig. 4 (2). One is the case that the analyzer dropped the response SYN+ACK segment. In this case, the SYN+ACK and ACK segments are exchanged in an actual sequence, and the 
Timing Error

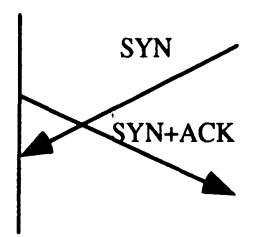

Drop SYN

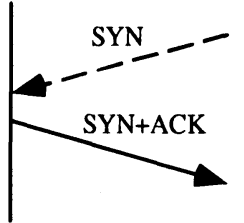

Actual Sequence

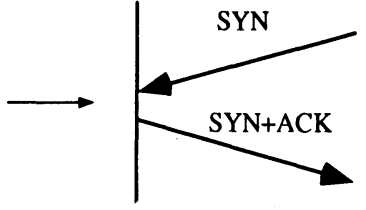

(1) Sending SYN+ACK in CLOSED

Drop SYN+ACK

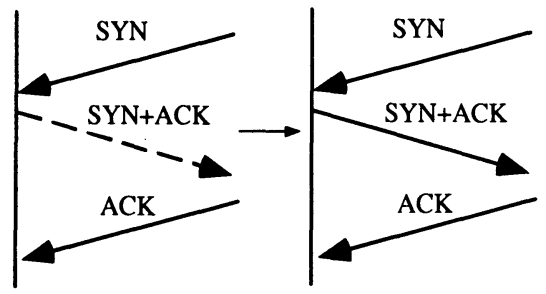

(2) Receiving SYN in CLOSED and No Response
Drop SYN+ACK

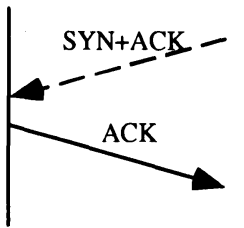

Detect SYN lost at Receiver Side

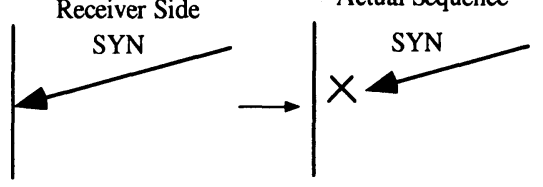

(3) Sending ACK in SYN-SENT
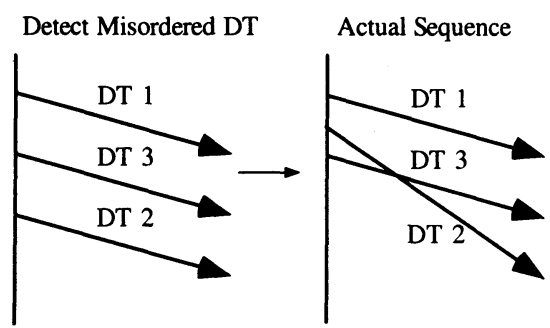

Drop DT or DT loss Actual Sequence

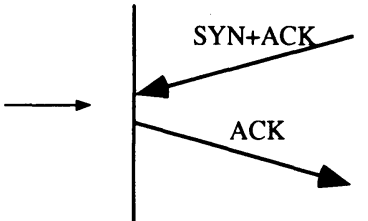

Actual Sequence
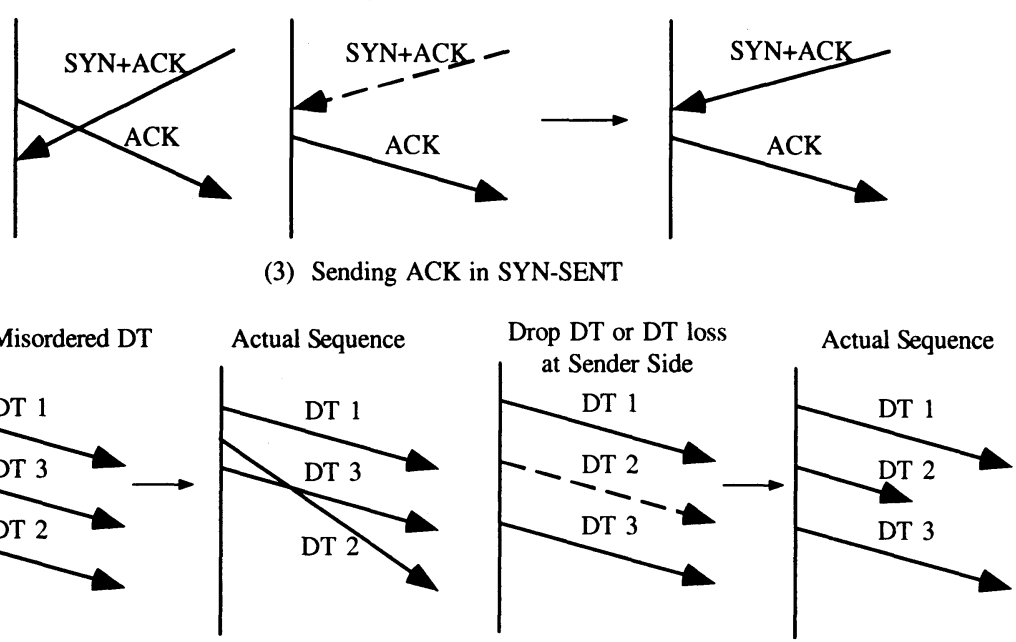

(4) Sending Out of Sequence DT in TRANSFER
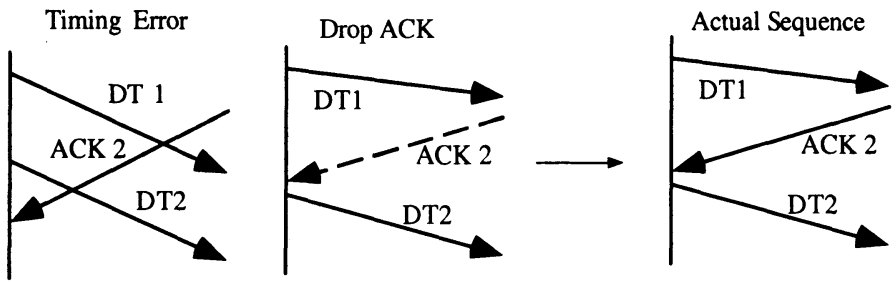

(5) Sending DT outside Upper Window Edge in TRANSFER

Figure 4 Exception Handling 
analyzer will detect the ACK segment. The other case is that the observed SYN segment was lost between the analyzer and the receiving computer. In this case, the SYN segment is not processed and the response to it is not returned. When the TCP emulation module detected this protocol error, it will search "ACK received" event. If it is found, the module considers that it will drop the SYN+ACK segment, and otherwise, the module considers that the SYN segment is lost in the network.

\section{(3) Sending ACK segment in state SYN-SENT}

This is the similar situation with (1) and there are two possibilities, timing error and dropping SYN+ACK segment. The TCP emulation module searches "SYN+ACK received" event. If it is found, the module considers that there are timing error, and otherwise, the module considers that it dropped a SYN+ACK segment.

(4) Sending DT segment whose sequence number is out of order in state TRANSFER

This is the case that the analyzer observed a DT segment (ACK segment which contains data) whose sequence number does not match snd_nxt which means the sequence number expected to be sent next. This case has two possibilities as depicted in Fig. 4 (4). One is that the analyzer has observed the misordered sequence of DT segments. The other is that the analyzer dropped the DT segment or it is lost between the analyzer and the sending computer. When the TCP emulation module detected this protocol error, it will search "DT received" event which contains the correct sequence number. If it is found, the module considers that there was misordering in the network and resequences the DT segments for the sending computer, and otherwise, the module considers that the DT segment is dropped or lost. In the latter case, snd_nxt is updated to match the subsequent DT segments.

(5) Sending DT segment whose sequence number is larger than upper window edge This is the case that the analyzer observed a DT segment whose sequence number plus data length exceeds the upper window edge. This is also similar with (1) and there are two possibilities. One is the case that there are timing errors for "ACK received" which opens the window and "DT sent" which violates the flow control mechanism. The other is the case that the analyzer has dropped the ACK segment which opens the window. In this case, the TCP emulation module searches "ACK received" which opens the window. If it is found, the module considers that there are timing error, and otherwise, the module considers that it dropped the ACK segment.

\section{RESULTS OF APPLYING TO ACTUAL WWW SERVER ACCESS}

In this section, we show the functions of our analyzer using results of applying it to an actual WWW server access. We have captured the communications between 
our local computer for a WWW client and the IFIP WWW server [7] in Austria. Figure 5 depicts the main window showing the emulation results. It shows the emulation result of individual computers, the left side for the client and the right side for the servers. For each event, it shows parameter values of PDUs of TCP and HTTP, the estimated values of state and variables of TCP and HTTP, and some comments concerning the protocol situation, together with the estimated time of each event.

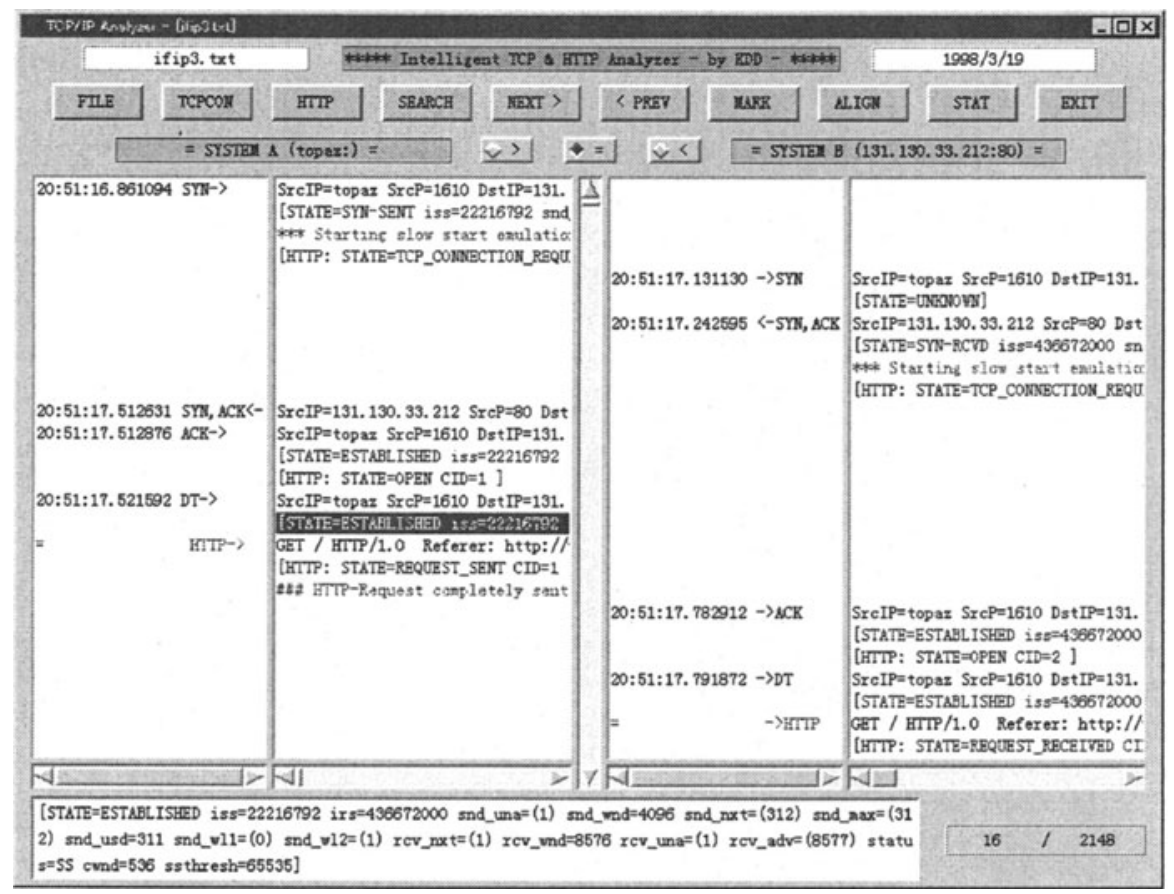

Figure 5 A Main window for Emulation Result

Although all of the analyzed information is included in this window, it would be difficult to understand because it shows the events corresponding to more than one TCP connections in a mixed way. Therefore, our analyzer prepares a window which shows the accessed Web page elements, the links among them, and the mapping between the Web page elements and TCP connections. Figure 6 depicts this window for the IFIP home page. The top home page "//www.ifip.or.at/" includes three image file as active links and has passive link to a Web page "//www.ifip.or.at/secr.htm." This window also gives the information of TCP connection, including IP address/host name, port number and the number of retransmitted TCP segments. Among them, the number of retransmitted TCP segments helps a network operator understand which TCP connection has problems. In the example of Fig. 6 , there are some retransmitted segments in the 
access to elements "//www.ifip.or.at/image/secr.gif."

“//www.ifip.or.at/image/ new.gif" and

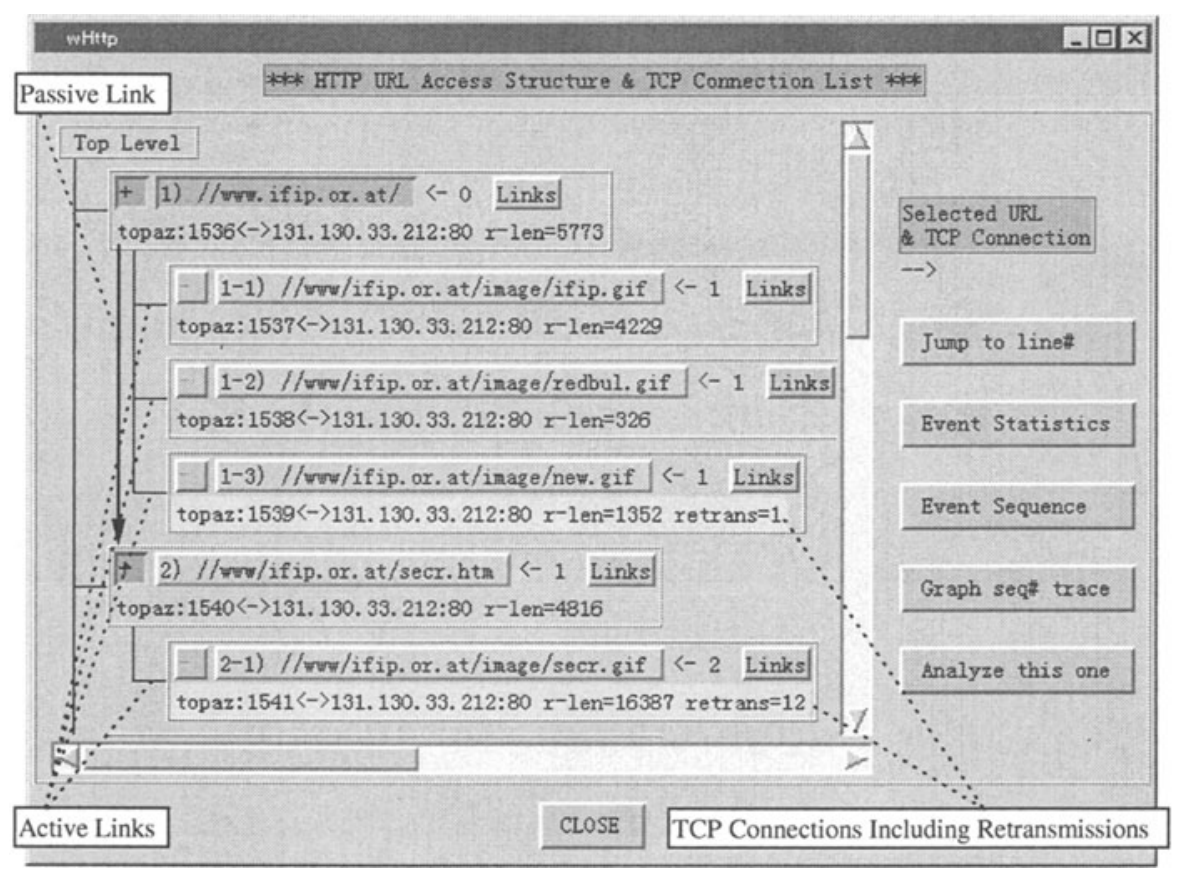

Figure 6 An Example of Web Page Links

By selecting one element, our analyzer shows the event sequence for the corresponding TCP connection. Figure 7 depicts a window showing an event sequence of the TCP connection transferring new.gif file. This window shows sending out and receiving of TCP segments and HTTP PDUs contained in TCP DT segments, in the style of sequence chart with the estimated time of sending out and receiving. As a TCP level information, this window also shows the relations between retransmitted TCP segments and its original segments, and the relations between DT segments and ACK segments.

In the case of Fig. 7, a SYN segment is retransmitted approximately 3 seconds after the first SYN segment is sent. It would be considered that the server had been too busy to respond the first request. 
** TCP \& HTTP Event Sequence Time Line *\$*

Relations between Original and Retransmitted Segment 190.33.212:80)

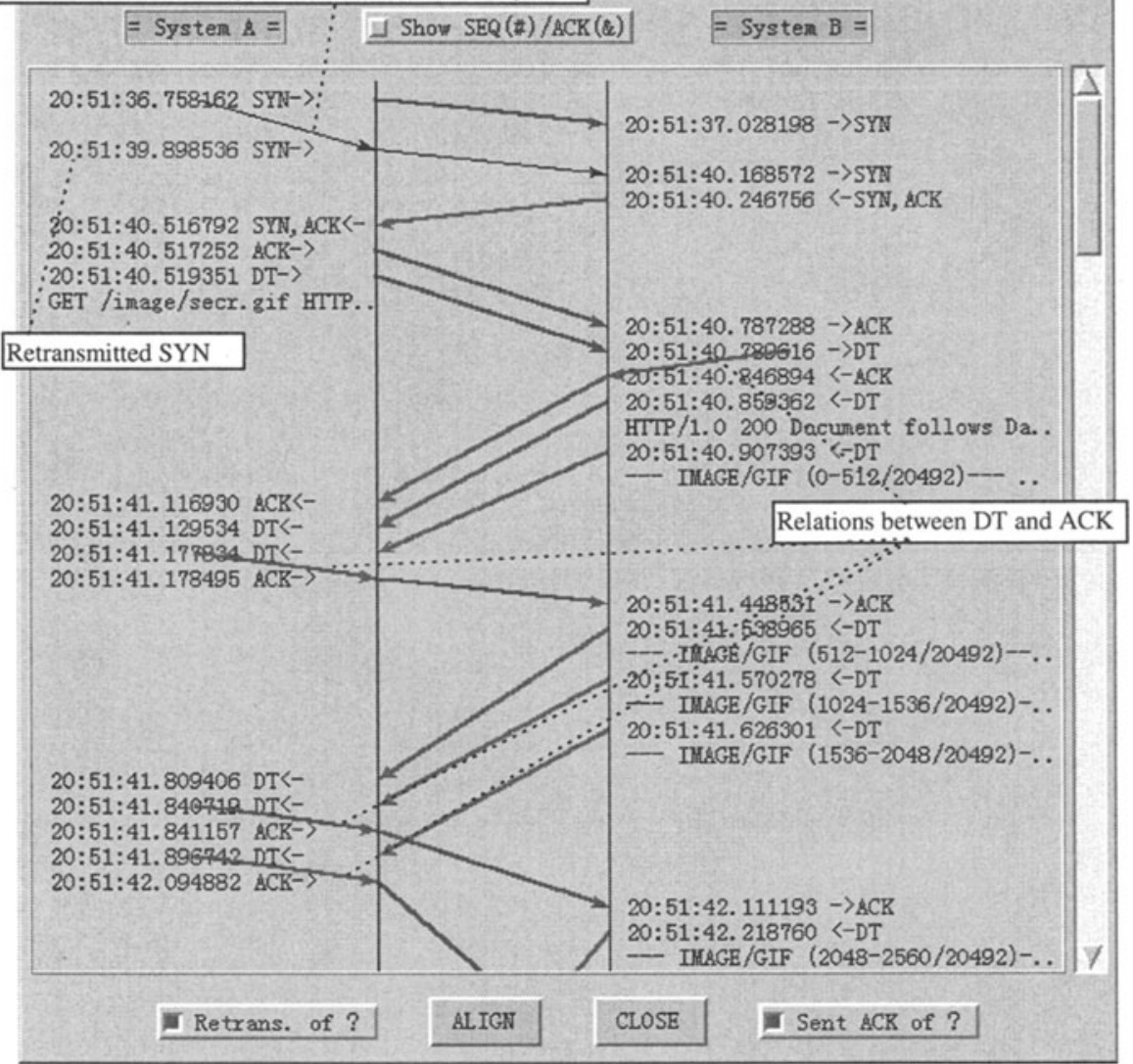

Figure 7 Example of Event Sequence Window

Figure 8 depicts an example of event sequence window, which includes retransmission of DT segments. In this case, it is considered that some data were retransmitted by fast retransmit mechanism because they were dropped in the network retransmit mechanism because they were dropped in the network. The functions showing the relations between DT and ACK segment and those between retransmitted segments and its original segments are effective in analyzing this case. Since the three ACK segments sent out by System A (the client) at 20:51:03.745422, :03.882042 and :03.893139 are duplicated ACK, System B (the server) retransmit the required DT segment according to the fast retransmit algorithm. The event sequence shows these explicitly. 


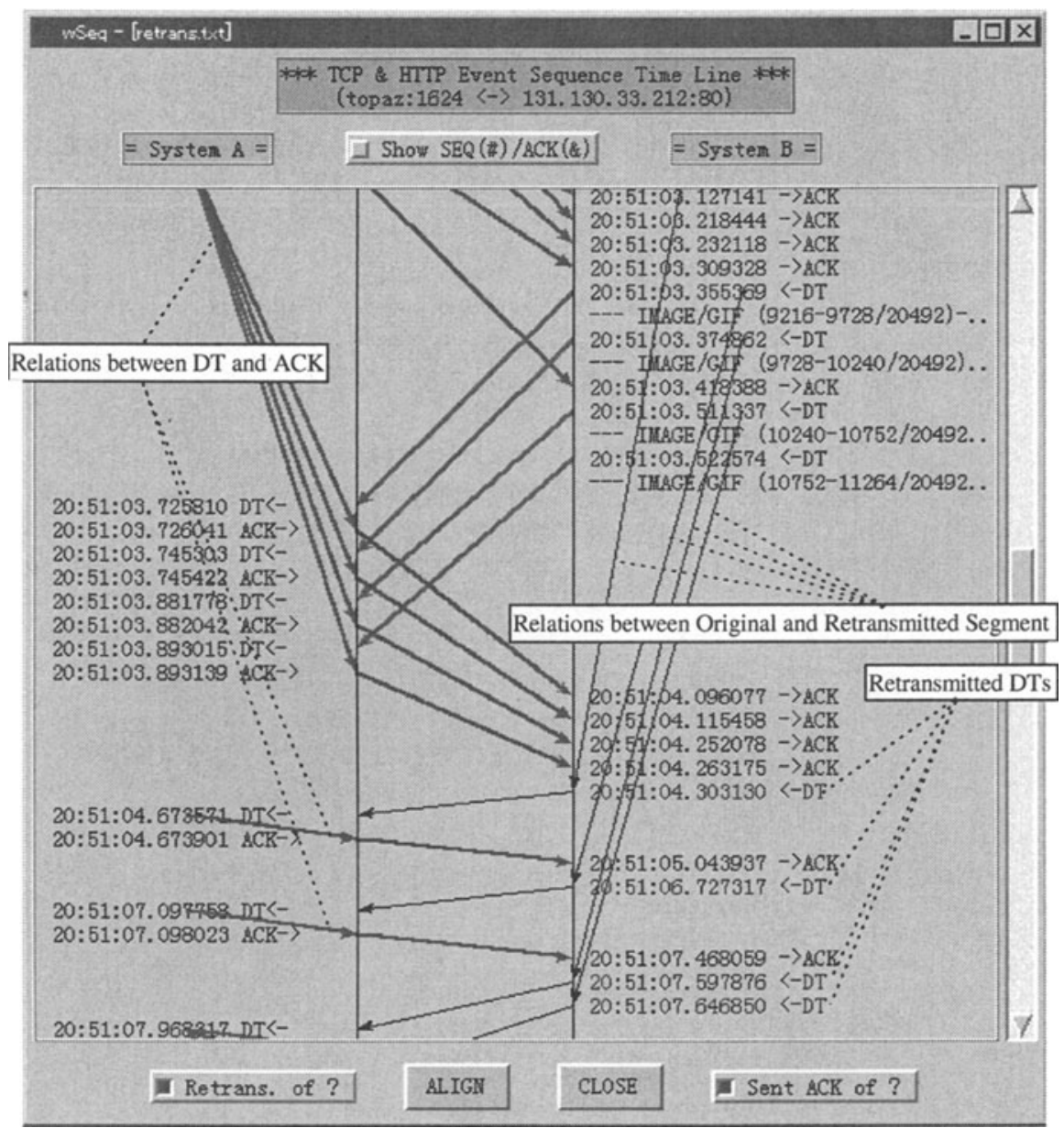

Figure 8 Example of Event Sequence Window with Retransmitted DT Segments

Our analyzer also has a function to show the transitions of the sequence number of transmitted segments, estimated congestion window (cwnd) and slow start threshold (ssthresh). Figure 9 shows those transitions for the TCP communication depicted in Fig. 8. In this case, there are some retransmission and therefore the cwnd is kept as a low value resulting in low throughput. This window is also helpful to analyzing the details of TCP level data transfer. 


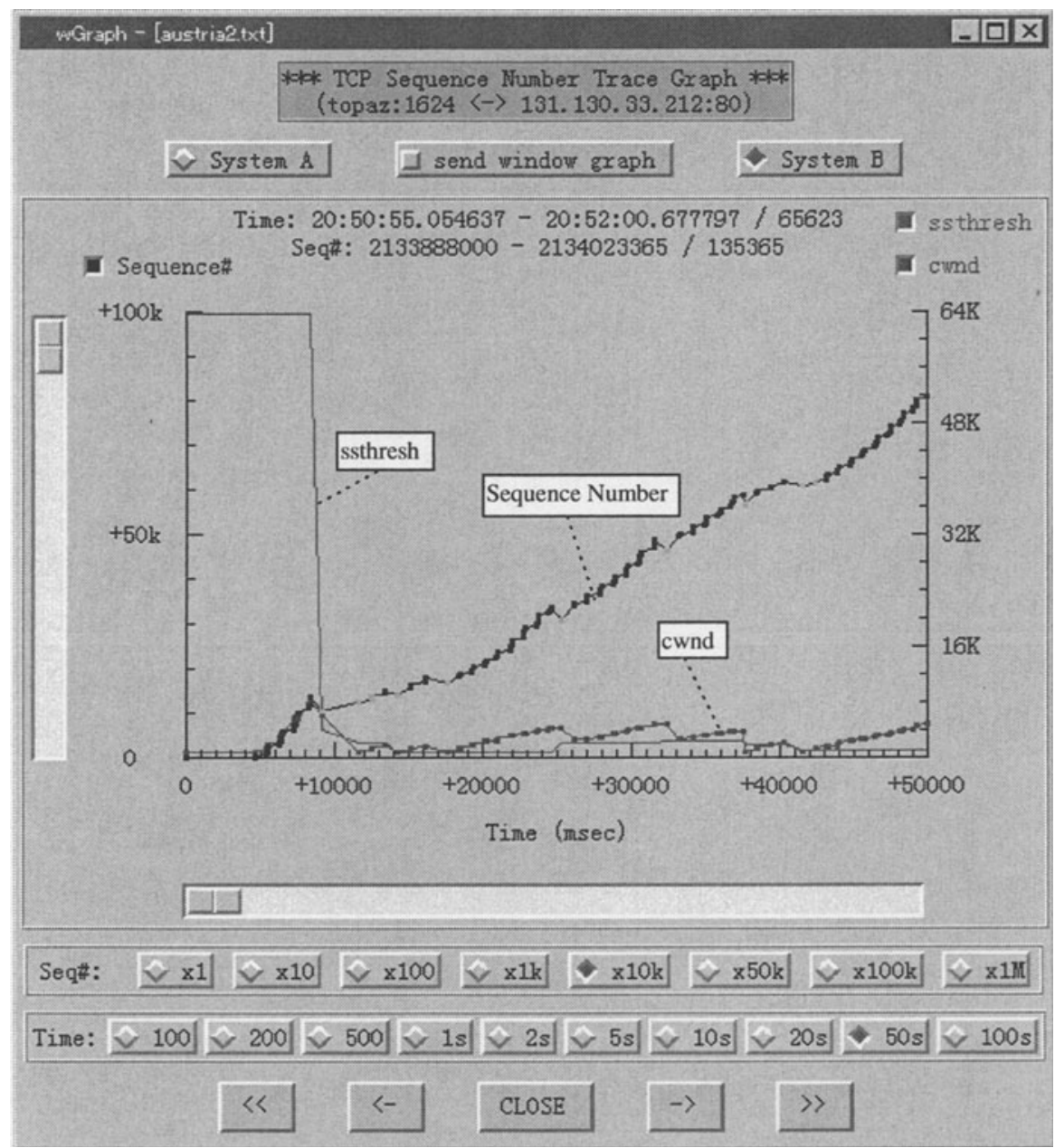

Figure 9 Transition of Sequence Number, Cwnd and Ssthresh of TCP Level

\section{CONCLUSION}

In this paper, we have described the design and examination results of our WWW intelligent protocol analyzer which can estimate what communication has taken place between WWW clients and servers focused on by emulating the behaviors of the IP, TCP and HTTP protocol entities in the clients and servers. The HTTP emulation function supports emulation of the HTTP state transition and estimation of Web page links by analyzing HTML text. This allows network operators to detect the structure of one Web page and the mapping between elements in the 
Web page and TCP connections. The TCP emulation function supports emulation of the TCP state transition and some internal procedures for the flow control such as the slow start algorithm. It can also handle exceptional situations such as monitoring errors and network misbehaviors.

This paper has also presented some results applying the analyzer to actual WWW server accesses. Our analyzer provides a window which show the links among Web page elements and the mapping among these elements and TCP connections. By selecting one element in this window, our analyzer can show an event sequence for TCP segments and HTTP PDUs and it can also show a transition of sequence number in TCP level. These graphical interfaces help network operators analyze the WWW server access in details.

\section{ACKNOWLEDGMENT}

The authors wish to thank Dr. T. Muratani, President of KDD R\&D Laboratories Inc., for his continuous encouragement to this study.

\section{REFERENCES}

[1] W. Richard Stevens, "TCP/IP Illustrated, Vol. 1 : The Protocols," Addison Wesley, 1994.

[2] DARPA Internet Program Protocol Specification, "Transmission Control Protocol," RFC 793, September 1981.

[3] T. Kato, T. Ogishi, A. Idoue and K. Suzuki, "Design of Protocol Monitor Emulating Behaviors of TCP/IP Protocols," Proc. of the International Workshop on Testing of Communication Systems, pp.416-431, September 1997.

[4] T. Kato, T. Ogishi, A. Idoue and K. Suzuki, "Intelligent Protocol Analyzer with TCP Behavior Emulation for Interoperability Testing of TCP/IP Protocols," Proc. of FORTE/PSTV, pp.449-464, November 1997.

[5] Tekelec, "Chameleon User's Manual," 1992.

[6] R. Fielding, et. al., "Hypertext Transfer Protocol -- HTTP/1.1," RFC 2068, January 1997.

[7] "http://www.ifip.or.at/," the URL of the IFIP WWW server

\section{BIOGRAPHY}

Tomohiko Ogishi is a research engineer of High Speed Communications Lab. in KDD R\&D Laboratories, Inc. Since joining KDD in 1992, he worked in the field of computer communication. His current research interests include the protocol testing on TCP/IP communication. He received the B.S. Degree of electrical engineering from the University of Tokyo in 1992. 
Akira Idoue is a senior research engineer of High Speed Communications Lab. in KDD R\&D Laboratories, Inc. Since joining KDD in 1986, he worked in the field of computer communication. His current research interests include implementation of high performance communication protocols and communication systems. He received the B.S. and M.E. Degrees of electrical engineering from Kobe University, Kobe, Japan, in 1984 and 1986 respectively.

Toshihiko Kato is the senior manager of High Speed Communications Lab. in KDD R\&D Laboratories, Inc. Since joining KDD in1983, he has been working in the field of OSI, formal specification and conformance testing, distributed processing, ATM and high speed protocols. He received the B.S., M.E. and Dr. Eng. Degrees of electrical engineering from the University of Tokyo, in 1978, 1980 and 1983 respectively. From 1987 to 1988, he was a visiting scientist at Carnegie Melon University. Since 1993, he has been a Guest Associate Professor of Graduate School of Information Systems, in the University of ElectroCommunications.

Kenji Suzuki is the executive vice president of KDD R\&D Laboratories, Inc. Since joining KDD in 1976, he worked in the field of computer communication. He received the B.S., M.E. and Dr. Eng. Degrees of electrical engineering from Waseda University, Tokyo, Japan, in 1969, 1972 and 1976 respectively. He received Maejima Award from Communications Association of Japan in 1988, Achievement Award from the Institute of Electronics, Information and Communication Engineers in 1993, and Commendation by the Minister of State for Science and Technology (Persons of scientific and technological research merit) in 1995. Since 1993, he has been a Guest Professor of Graduate School of Information Systems, in the University of Electro-Communications. 